\title{
Etablierung von Hochdurchsatz-Kultivierungs- und -Screeningmethoden für phototrophe Einzeller
}

\author{
Ulrich M. Tillich, Stefanie Grüber, Marcus Frohme
}

Zusammenfassung

Mit Hochdurchsatz-Kultivierungs- und -Screeningmethoden können viele Proben parallel, miniaturisiert und kostengünstig bearbeitet werden. Für phototrophe Organismen wie Mikroalgen und Cyanobakterien sind Hochdurchsatz-Kultivierungsverfahren jedoch bis heute kaum etabliert. Im Rahmen dieser Arbeit wurden diese Verfahren beispielhaft für das Cyanobakterium Synechocystis sp. PCC 6803 etabliert. Die benötigte technische Automatisierung wurde hierbei durch den Einsatz eines Tecan Genesis RSP 150 Pipettierroboters erreicht. Die Kultivierung erfolgte in Deepwell-Mikrotiterplatten innerhalb einer speziell angefertigten Kammer mit programmierbaren Schüttlern, einstellbarer Belichtung und $\mathrm{CO}_{2}$-Atmosphäre. Die in diesem System erreichten Wachstumsraten sind vergleichbar mit publizierten Kultivierungsmethoden. Das Hochdurchsatz-Screening wurde mit Hilfe eines in den Roboter integrierten Tecan Genios Plus Plattenreaders durchgeführt. Es wurden beispielhaft Methoden zur Bestimmung von optischer Dichte und Chlorophyllgehalt etabliert. Die hier vorgestellte Plattform kann vielseitig zur Analyse phototropher Organismen eingesetzt werden und ist durch entsprechende Assays leicht zur Messung anderer Parameter erweiterbar.

\section{Abstract}

High-throughput cultivation and screening methods allow a parallel, miniaturized and cost efficient processing of many samples. These methods however, have not been generally established for phototrophic organisms such as microalgae or cyanobacteria. In this work we describe and test high-throughput methods with the model organism Synechocystis sp. PC6803. The required technical automation for these processes was archived with a Tecan Genesis RSP 150 pipetting robot. The cultivation was performed in deepwell microtiter plates within a specially constructed cultivation chamber. The chamber is outfitted with programmable shaking conditions, variable illumination and an adjustable $\mathrm{CO}_{2}$ atmosphere. The growth rates archived within this system are comparable to those achieved with established methods such as bioreactors. The high-throughput screening was achieved with a Tecan Genios Plus plate reader integrated within the pipetting robot. Methods for determination of optical density and amount of chlorophyll were established within the scope of this work. The presented platform can be used for a variety of analyses of phototrophic organisms and is easily expandable with further assays to screen for additional targets.

\section{Einleitung}

Hochdurchsatz-Kultivierung und -Screening sind nur mit einer Miniaturisierung der Kultur und Parallelisierung der Prozesse über den Einsatz von Mikrotiterplatten möglich (Fernandes/Cabral 2006; Mayr/Fuerst 2008). Hierbei handelt es sich um rechteckige Kunststoffplatten mit voneinander getrennten Kavitäten (sogenannten Wells), welche als Reaktionsräume dienen. Mikrotiterplatten haben verschiedene Formate mit unterschiedlicher Anzahl (und Volumina) der Wells. Zu Beginn der 90er Jahre wurden 96-Well-Formate entwickelt, sodass 96 Proben parallel bearbeitet werden konnten. Durch weitere Miniaturisierungen konnte bis heute der Durchsatz auf 3456 Wells pro Platte gesteigert werden. Besonders Deepwell-Mikrotiterplatten mit einem Volumen von bis zu $5 \mathrm{ml}$ finden in der Kultivierung von Mikroorganismen Anwendung (Kumar et al. 2004). Abbildung 1 zeigt eine Deepwell-Mikrotiterplatte, welche für die Kultivierung von Cyanobakterien eingesetzt wird.

Die Mikrotiterplatte wurde zwischen 1980 und 1990 erstmals für Untersuchungen zum Wachstum mikrobieller Kulturen eingesetzt. Sowohl das Wissen, als auch die Technologien sind in diesem Bereich seither rapide gewachsen. Heute stehen weitere technische Entwick- 


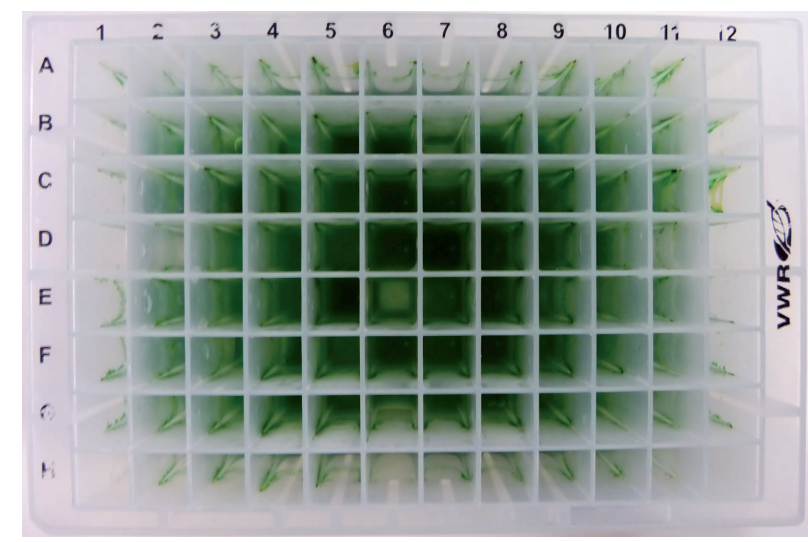

Abb. 1: Kultivierung in Mikrotiterplatte. Mit Hilfe einer in den Pipettierroboter Tecan Genesis RSP 150 integrierten Kultivierungskammer erfolgt im Labor für Molekularbiologie und Funktionelle Genomik die miniaturisierte Kultivierung von Cyanobakterien in 96-Deepwell-Mikrotiterplatten.

lungen wie Multipipetten, Pipettierroboter, Plattenreader und Autosampler zur Verfügung (Duetz 2007).

Die Hochdurchsatz-Kultivierung in Mikrotiterplatten wird routinemäßig für den bakteriellen Modellorganismus E.coli eingesetzt (Huber et al. 2010). Für photothrophe Organismen sind Verfahren der Hochdurchsatz-Kultivierung bis heute jedoch kaum etab- liert. Hochdurchsatz-Screening findet erst seit Anfang der 90er Jahre Anwendung. Besonders im Bereich der modernen Wirkstoffsuche in Pharma- sowie Biotechnologie-Unternehmen stellt es die Basis sowohl für die Grundlagen- als auch für die angewandte Forschung dar. Ohne die massiv parallele Bearbeitung von Proben, die durch das Hochdurchsatz-Screening ermöglicht werden, wären die schnellen Fortschritte in diesen Gebieten kaum möglich.

Als Hochdurchsatz-Screening werden hierbei Verfahren bezeichnet, welche einen Durchsatz von 10.000 bis 100.000 Untersuchungen pro Tag erreichen. Voraussetzungen hierfür sind die Automatisierung der Prozesse bis hin zu vollautomatisierten Robotersystemen (Carroll et al. 2004; Wölcke et al. 2001). Im Labor für Molekularbiologie der TH Wildau wird an Methoden der Hochdurchsatz-Kultivierung und des HochdurchsatzScreenings einzelliger Mikroalgen und Cyanobakterien gearbeitet. Im Rahmen dieser Arbeit wurden diese Methoden beispielhaft für das Cyanobacterium Synechocystis sp. PCC 6803 etabliert. Das integrierte Verfahren kann vielseitig eingesetzt werden, um z. B. biotechnologisch relevante Algenstämme zu identifizieren.

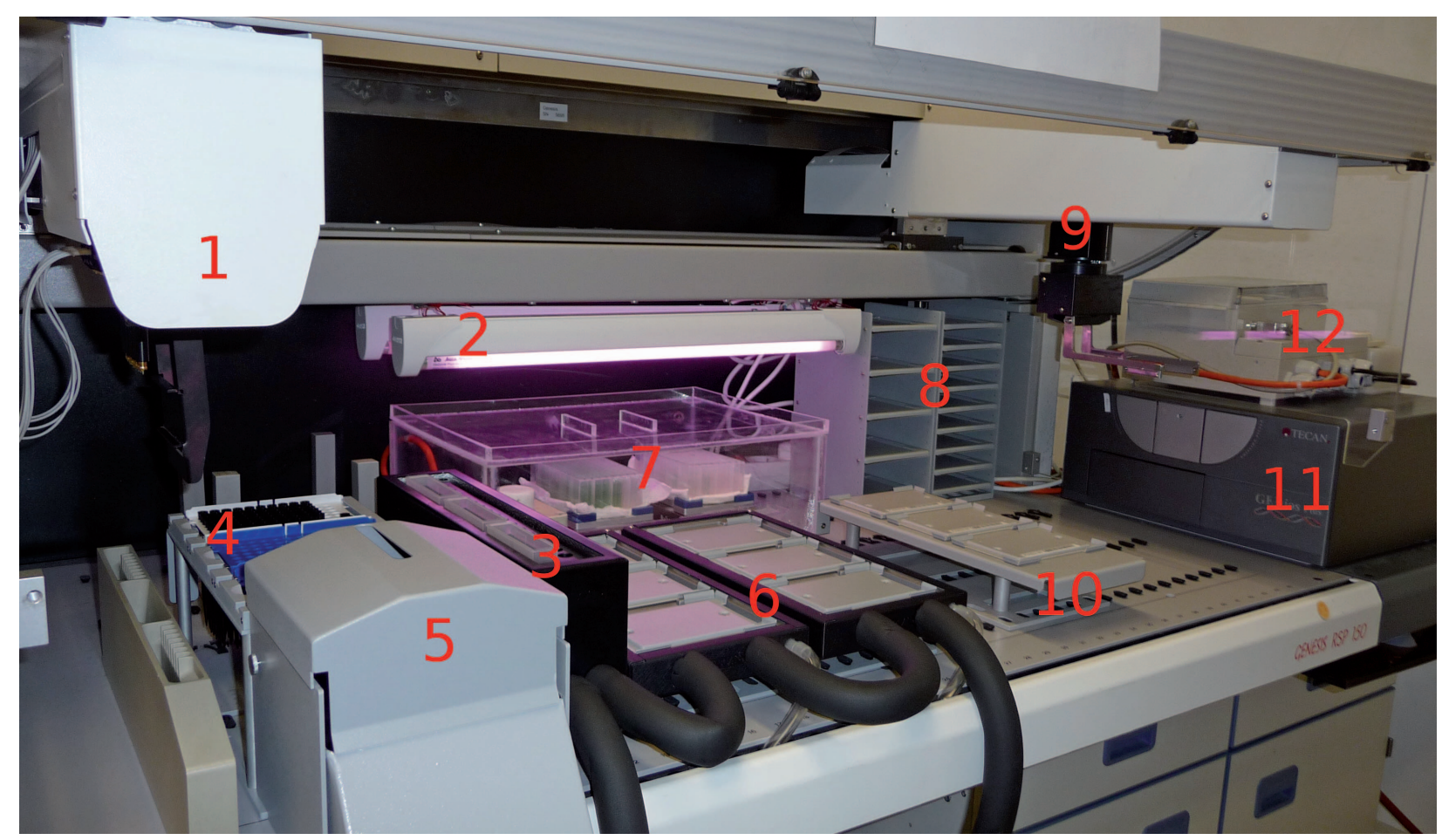

Abb. 2: Tecan Genesis RSP 150 Pipettierroboter mit integriertem Tecan GENios Plus Plattenreader sowie einer Algenkultivierungkammer mit CO ${ }_{2}^{-}$ Regulierung via Magnetventil.

[1] Liquid-Handling-Arm (LiHa), [2] Stufenlos steuerbare Lichtquelle (3,53- 83,39 $\mu \mathrm{E} / \mathrm{m}^{2}$ ), [3] Temperierbare Racks (Ablagegestelle) für Reagenzien, [4] Rack für Pipettenspitzen, [5] Abwurfbehälter für Pipettenspitzen, [6] Temperierbare Racks für Mikrotiterplatten, [7] Kultivierungskammer für Algen mit 2 Schüttlerplätzen, [8] Hotel (zur Lagerung von Mikrotiterplatten), [9] Robotic-Manipulator-Arm (RoMa), [10] Rack für Mikrotiterplatten,

[11] Plattenreader Tecan GENios Plus, [12] Steuereinheit für die Kultivierungskammer ( $\mathrm{CO}_{2}$ und Lichtintensität) 


\section{Material und Methoden}

\section{Aufbau der Hochdurchsatzplattform}

Für Anwendungen im Hochdurchsatz sind einige technische Voraussetzungen zwingend erforderlich: Die Automatisierung der Kultivierungs- und der Detektionstechniken wurden im Labor für Molekularbiologie und Funktionelle Genomik der TH Wildau durch den Einsatz eines Tecan Genesis RSP 150 Pipettierroboters, erreicht (Abb. 2).

Der Pipettierroboter Tecan Genesis RSP 150 ist eine automatisierte Pipettierstation und gut für den Hochdurchsatz geeignet. Neben einem Liquid-HandlingArm (LiHa) zum Pipettieren von Flüssigkeiten mit hoher Reproduzierbarkeit verfügt er zusätzlich über einen Robotic-Manipulator-Arm (RoMa). Dieser ermöglicht das Bewegen von Objekten innerhalb des Robotersystems. Sowohl Mikrotiterplatten als auch Reagenzien können durch temperierte Racks (Ablagegestellen) auf bis zu $0{ }^{\circ} \mathrm{C}$ gekühlt werden.

Der Pipettierroboter ist mit einer Kultivierungskammer ausgestattet (Abb. 2 Nr. 7), welche an der TH Wildau speziell für die Anforderungen von photothrophen Organismen konstruiert wurde. Sie ermöglicht eine vollständige automatisierte Kultivierung innerhalb des Roboters bei geringer Kontaminationsgefahr. Die Kultivierungskammer ist komplett über den RoMa ansteuerbar. Der Roboterarm ist in der Lage, den Deckel zu entfernen und die Kultivierungsplatten auf ein Rack zu überführen, wo sie anschließend von dem LiHa-Arm erreicht werden können.

In der Kammer stehen zwei computergesteuerte Schüttlerplätze für die Kultivierung in Mikrotiterplatten zur Verfügung. Die Einstellungen der beiden Schüttler sind individuell vom PC aus konfigurierbar, sodass auch komplexere Bewegungen programmiert werden können. Die Kultivierungskammer verfügt außerdem über eine $\mathrm{CO}_{2}$-Versorgung, welche eine Regelung der Atmosphäre in der Kammer von 0 bis $4 \% \mathrm{CO}_{2}$ ermöglicht. Bei Unterschreiten des Sollwertes (gemessen durch einen in der Kultivierungskammer befindlichen Sensor) wird über eine Steuereinheit ein Magnetventil geöffnet und $5 \%$ - $\mathrm{CO}_{2}$-Gas-Gemisch hinzu dosiert, bis der Sollwert wieder erreicht ist. Darüber hinaus besteht die Möglichkeit einer stufenlosen Regelung der Lichtintensität von $3,53-83,39 \mu \mathrm{E} / \mathrm{m}^{2}$.

Durch den im Robotersystem integrierten Plattenreader Tecan GENios Plus (Abb. 2 Nr. 11) werden automatisierte Absorptions- und Fluoreszenzmessungen ermöglicht. Neben direkten optischen Parametern, wie der Lichtstreuung bei $750 \mathrm{~nm}$ zur Zellzahlbestimmung oder der Absorption bei $650 \mathrm{~nm}$ für extrahierte Chlorophyllpigmente, können durch das Einsetzen von entsprechenden Assays eine Vielzahl von verschiedenen Analyten detektiert werden. So kann beispielsweise durch den Einsatz eines ELISA's (Enzyme-linked Immunosorbent Assay) fast jeder beliebige Analyt detektiert und quantifiziert werden.

\section{Kultivierung in Mikrotiterplatten}

Nach einer Kultivierungszeit von 24 Tagen auf BG11Nähragarplatten (BG11, 1 \% Agar) wurden die vereinzelten Cyanobakterien-Kolonien von den Agarplatten entnommen und in 96-Deepwell-Mikrotiterplatten überführt (Abb. 1). Die Kultivierung erfolgte in marinem BG11-Nährmedium (42 g/l Instant Ocean, 17,65 mM NaNO3, 0,18 mM K2HPO4 * 3H2O, 0,03 mM Citronensäure, 0,003 mM EDTA, 0,19 mM Na2CO3, 0,03 mM Ammoniumeisen(III)-citrat und Spurenelemente).

In Vorversuchen hat sich gezeigt, dass für die Kultivierung von Synechocystis sp. PCC 6803 ein Schüttelprotokoll von je 5 Bewegungen Nord-Süd und 5 Bewegungen Ost-West bei 800 rpm optimal ist, um die Kulturen in der Lösung zu halten. Die Atmosphäre in der Kammer war hierbei konstant auf $2 \% \mathrm{CO}_{2}$ eingestellt. Die Belichtung wurde der Dichte der Kultur angepasst und betrug zu Anfang der Kultivierung 3,53 $\mathrm{\mu E} / \mathrm{m}^{2}$. Sie wurde stufenweise wie folgt angehoben: $3,66 \mu \mathrm{E} / \mathrm{m}^{2}$ (3. Tag); $5,83 \mu \mathrm{E} / \mathrm{m}^{2}$ (4. Tag); 10,75 $\mu \mathrm{E} / \mathrm{m}^{2}$ (7. Tag). Als Kontrolle für Kreuzkontamination zwischen benachbarten Wells wurden einzelne Wells lediglich mit Nährmedium befüllt, aber nicht mit gepickten Kolonien angeimpft.

Im Verlauf der Kultivierung war eine wöchentliche Auffüllung der einzelnen Wells mit BG11-Nährmedium erforderlich, um das evaporierte Medium zu ersetzen. Hierbei wurden je ein Milliliter Kultur mit einem Milliliter BG11 gemischt (1:2 Verdünnung). Ebenso erfolgte eine tägliche Resuspension der Zellen über die Pipettierfunktion des Roboters, da ansonsten trotz der optimalen Schüttellbedingungen nach über 24 Stunden ein Absetzen der Zellen am Grund der Wells zu beobachten war.

\section{Messung der optischen Dichte}

Die optische Dichte bei $750 \mathrm{~nm}\left(\mathrm{OD}_{750}\right)$ wird bei Mikroalgen und Cyanobakterien zur Bestimmung der Zellzahl verwendet. Bei dieser Wellenlänge absorbieren die Zellen das Licht nur minimal, demnach ist die 
gemessene Lichtabnahme durch Streuung der Zellen in der Suspension (gegen Nährmedium als Blindwert) der Zellzahl proportional. Die $\mathrm{OD}_{750}$ wurde für alle Wells täglich gemessen. Hierfür wurden die jeweiligen Kulturen zuvor durch auf- und abpipettieren resuspendiert. Anschließend erfolgte eine Überführung von 80jl je Well in eine transparente Mikrotiterplatte mit 384 Wells. Hierbei wurden jeweils eine Spalte sowie eine Reihe nicht belegt, um mögliche Streueffekte ausschließen zu können.

\section{Chlorophyllbestimmung}

Für die Bestimmung des Gesamtchlorophyllgehaltes in den Zellen wurden je Messung 300 pl Probenvolumen je Well verwendet. Die Zellen wurden bei $3.320 \mathrm{~g}$ und $9{ }^{\circ} \mathrm{C}$ für 15 min mit Hilfe einer Plattenzentrifuge vom Nährmedium getrennt. Nach Entfernung von $270 \mu \mathrm{l}$ Überstand erfolgte die Resuspension in den verbleibenden $30 \mu \mathrm{l}$ mittels Plattenshaker für 15min (800 rpm, $\left.5{ }^{*} \mathrm{~ns}, 5^{*} \mathrm{Ow}\right)$. Durch die Zugabe von 270 pl Methanol (100\%) und einer anschließenden Inkubationszeit von einer Stunde im Kühlschrank $\left(4^{\circ} \mathrm{C}\right)$ wurden die Chlorophylle aus ihrer natürlichen proteinogenen Umgebung extrahiert. Die Proben wurden im Anschluss erneut für $15 \mathrm{~min}$ bei $3.320 \mathrm{~g}$ und $9{ }^{\circ} \mathrm{C}$ zentrifugiert. Die Messung der Absorption des Überstandes erfolgte in Doppelbestimmung bei 650nm gegen Methanol unter Verwendung des Plattenreaders (384-Well-Platte mit $80 \mu l$ je Well). Der Chlorophyllgehalt ergibt sich durch folgende Formel:

Chlorophyll $[\mu \mathrm{g} / \mathrm{ml}]=\frac{\text { Absorption }_{650 \mathrm{~nm}} * 13,38}{\text { Probenvolumen }[\mathrm{ml}]}$

Der Extinktionsfaktor von 13,38 wurde anhand von Proben mit bekannter Chlorophyllkonzentration empirisch ermittelt.

\section{Wachstumsrate}

Die spezifische Wachstumsrate $\mu\left[\mathrm{d}^{-1}\right]$ bezeichnet die Wachstumsgeschwindigkeit zum Zeitpunkt $\mathrm{t}$ für einen definierten Organismus unter bestimmten Bedingungen (pH-Wert, Temperatur, Nährmedium usw.).

Die Ermittlung erfolgte unter Verwendung nachfolgender Gleichung (Csögör 2000):

$\boldsymbol{\mu}=\frac{\ln \left(\mathrm{x}_{t}\right)-\ln \left(\mathrm{x}_{0}\right)}{\Delta t}$

$\boldsymbol{\mu}$ Spezifische Wachstumsgeschwindigkeit $\left[\mathrm{d}^{-1}\right]$

$\Delta t$ Änderung der Zeit [d]

$\left(\mathrm{x}_{t}\right)$ Optische Dichte zum Zeitpunkt $\mathrm{t}$

$\left(\mathrm{x}_{0}\right)$ Optische Dichte zum Zeitpunkt $\mathrm{t}=0$

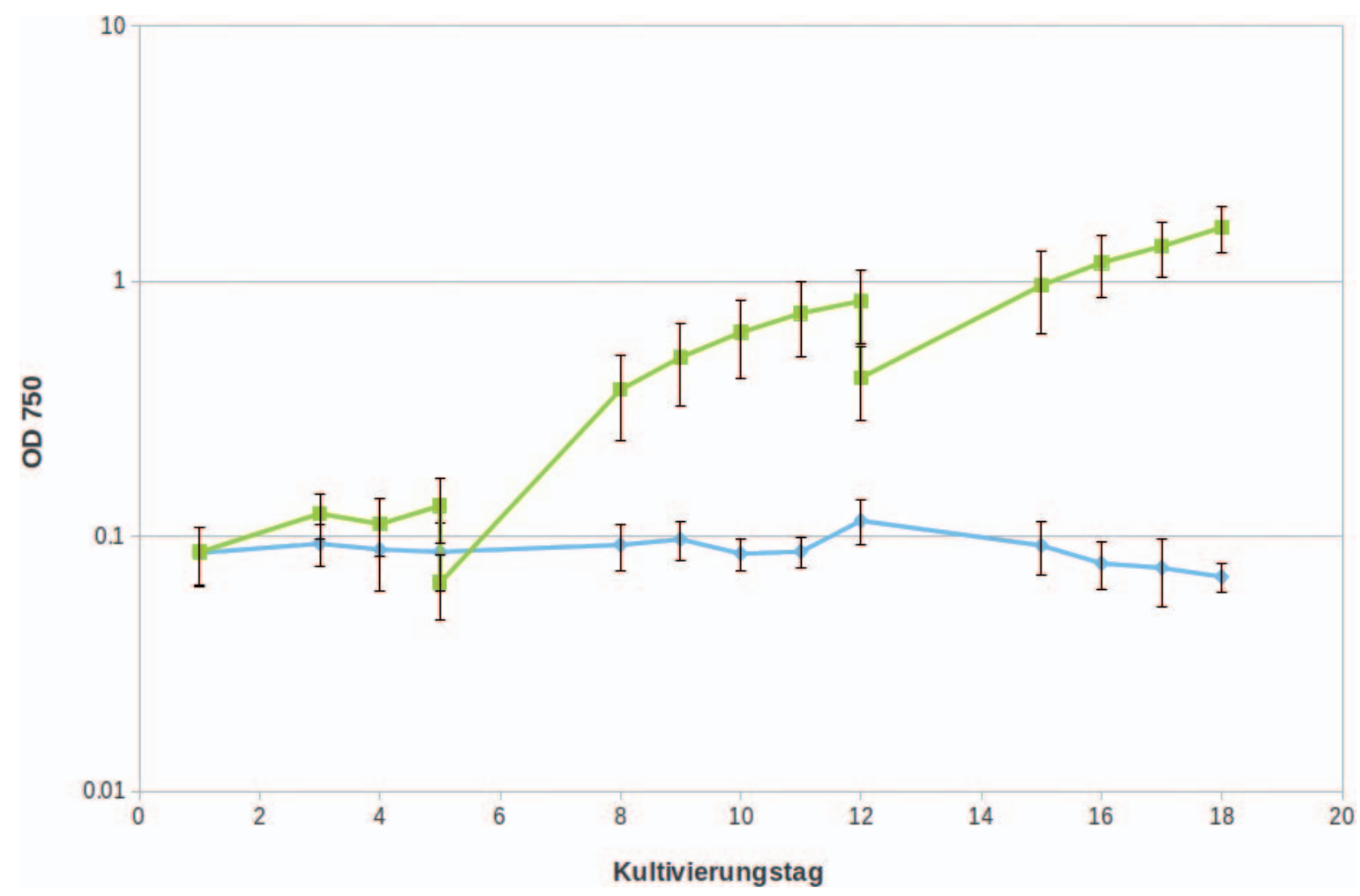

Abb. 3: $\mathrm{OD}_{750}$ über der Kultivierungszeit: Angeimpfte Wells (grün) im Vergleich mit Kontroll-Wells (blau). An den Tagen 5 und 12 wurde $1: 2$ mit Medium verdünnt. 


\section{Berechnung der Generationszeit}

Unter der Generationszeit $\left[{ }^{t} d\right]$ wird die Zeit verstanden, in der sich die Zahl der Individuen einer Population von Lebewesen verdoppelt. Während der exponentiellen Phase ist die Generationszeit am kürzesten. Das bedeutet, dass sich die Wachstumsgeschwindigkeit in den einzelnen Wachstumsphasen unterscheidet. Demnach kann die Generationszeit mit der Wachstumsgeschwindigkeit nicht gleichgesetzt werden. Die Wachstumsrate lässt sich mit Hilfe der ermittelten durchschnittlichen Wachstumsrate $\mu$ wie folgt berechnen:

$t_{d}=\frac{\ln (2)}{\mu}$

\section{Ergebnisse}

Mit den in Vorversuchen etablierten Parametern und Methoden wurde ein voller Kultivierungslauf mit dem Modellorganismus Synechocystis sp. PCC 6803 durchgeführt. Die Temperatur innerhalb der Kammer wurde im Verlauf des Wachstums beobachtet und lag zwischen 25 bis $27^{\circ} \mathrm{C}$. In Abbildung 3 ist das Wachstum der Zellen in den einzelnen Wells dargestellt. Es ist klar zu erkennen, dass bei den angeimpften Wells ein gutes Wachstum stattgefunden hat. In den ersten 5 Tagen hat kaum Wachstum stattgefunden, nach der ersten Verdünnung war jedoch eine starke Zunahme der OD von Tag zu Tag zu beobachten. Die Streuung der OD-Werte zwischen den Wells ist anhand der Standardabweichungsbalken ebenfalls gut zu erkennen. In den Kontroll-Wells hingegen war kein Wachstum messbar. Vorherige Kultivierungen (Daten nicht gezeigt) haben ähnliche Wachstumsverläufe gezeigt. Die Wachstumsraten und Generationszeiten wurden für Kultivierungszeiträume zwischen den Verdünnungen bestimmt und sind in Tabelle 1 aufgelistet.

Am Tag 18 wurde für alle Wells der Chlorophyllgehalt bestimmt. Im Durchschnitt ergab sich für die Wells

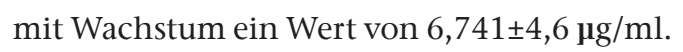

\begin{tabular}{|c|c|c|}
\hline $\begin{array}{l}\text { Kultivierungs- } \\
\text { zeitraum }\end{array}$ & Wachstumsrate $\mu\left[\mathrm{d}^{-1}\right]$ & Generationszeit $\left[\mathrm{t}_{\mathrm{d}}\right]$ \\
\hline Tag 1-5 & $0,084 \pm 0,047$ & $12,265 \pm 9,114$ \\
\hline Tag 5-12 & $0,353 \pm 0,066$ & $2,048 \pm 0,505$ \\
\hline Tag 12-18 & $0,235 \pm 0,045$ & $3,049 \pm 0,564$ \\
\hline
\end{tabular}

Tab. 1: Durchschnittliche Wachstumsraten und Generationszeiten für die Wells mit OD-Zunahme

\section{Diskussion}

Der Kultivierungslauf hat gezeigt, dass der Aufbau der Hochdurchsatzplattform für Anwendungen mit phototrophen Einzellern gut geeignet ist. Eine parallele Kultivierung der Cyanobakterien in Mikrotiterplatten innerhalb der Kultivierungskammer konnte erfolgreich durchgeführt werden und die neu etablierten Methoden zeigten konsistente und reproduzierbare Daten.

Die $\mathrm{OD}_{750}$-Werte in den ersten 5 Tagen der Kultivierung zeigten eine deutliche lag-Phase, in der die Kulturen noch nicht stark gewachsen sind, da sie sich erst an die Umgebung in der Flüssigkultur anpassen mussten. In dieser Phase war ebenso die Wachstumsrate mit $\boldsymbol{\mu}=0,084$ sehr niedrig. Nach den ersten 5 Kultivierungstagen war jedoch eine starke, fast exponentielle Wachstumszunahme an der OD zu erkennen (log-Phase). Die OD-Werte zwischen den Wells zeigten in dieser Phase eine große Standardabweichung. Dies ist nicht auf stark unterschiedliche Wachstumsraten zurückzuführen, sondern darauf, dass die Kulturen in den einzelnen Wells mit unterschiedlichen Start-ODs in die log-Phase übergingen, aber trotzdem ähnlich schnell gewachsen sind. Die hohen, in der log-Phase erreichten Wachstumsraten 0,353 $\pm 0,066$ und 0,235 $\pm 0,045$, sind mit anderen Kultivierungsmethoden (z. B. in Bioreaktoren) vergleichbar und zeigen, dass die im Roboter erzeugten Bedingungen gut für die Kultivierung geeignet sind. Die

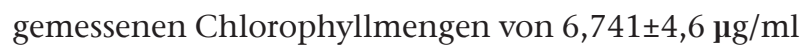
deuten außerdem auf eine gute Pigmentierung der Zellen hin, was für eine hohe Vitalität spricht.

In den Kontroll-Wells, welche nur mit Medium befüllt waren, war kein Wachstum von Zellen detektierbar. Bereits das Übertreten weniger Zellen hätte jedoch zu einer (verzögerten) Zunahme der OD geführt. Somit ist gezeigt, dass keine Kreuzkontamination zwischen Wells durch das Schütteln oder die Probenentnahme erfolgt ist und dass jedes Well als eine eigene, vollständig getrennte Kultivierungseinheit betrachtet werden kann.

\section{Fazit}

Hochdurchsatz-Kultivierungs- und -Screeningmethoden für phototrophe Organismen konnten erfolgreich etabliert werden. Die miniaturisierte und parallele Kultivierung in Mikrotiterplatten erlaubt es, bis zu 192 Kulturen gleichzeitig und automatisiert im Roboter zu 
kultivieren. Die für das Hochdurchsatzscreening getesteten Meßmethoden waren ebenfalls erfolgreich und können mit dem integrierten Plattenreader und entsprechenden Assays leicht auf weitere Parameter oder Analyten ausgeweitet werden.

\section{Danksagung}

Unser besonderer Dank gilt der Cyano Biofuels GmbH für die gute Zusammenarbeit und die finanzielle Unterstützung.

Unser Dank gilt weiterhin Sven Angermann, Andreas Frahm und Kai Lietzau für die Unterstützung beim Bau der Kultivierungskammer.

\section{Literatur}

Carroll, S., Inglese, J., Mao, S., Olsen, D. (2004): In Molecular Cancer Therapeutics: Strategies for Drug Discovery and Development. John Wiley \& Sons, Inc. USA, 119-140.

Doig, S., Pickering, S., Lye, G., Baganz, F. (2005): Modelling surface aeration rates in shaken microtitre plates using dimensionless groups. Chemical Engineering Science, Vol. 60 Nr. 10, United Kingdom, 2741-2750.

Duetz, W. (2007): Microtiter plates as mini-bioreactors: miniaturization of fermentation methods. Trends in Microbiology, Vol.15 Nr.10, Niederlande, 469-475.

Fernandes, P., Cabral, JMS. (2006): Microlitre/mililitre shaken bioreactors in fermentative and biotransformation process. Biocatalysis and Biotransformation, Vol. 24 Nr. 4, Portugal, 237-252.

Csögör, Z. (2000): Untersuchung zur Modellierung des Wachstums und der Produktbildung bei Mikroalgen. Dissertation, Shaker Verlag, Deutschland, 52.

Hermann, R., Lehmann, M., Büchs, J. (2003): Characterization of gasliquid mass transfer phenomena in microtiter plates. Biotechnology and Bioengineering, Vol. 81 Nr. 2, Deutschland, 178-186.

Huber, R., Palmen, T., Ryk, N., Hillmer, A., Luft, K., Kensy, F., Büchs, J. (2010): Replication methods and tools in high-throughput cultivation process - recognizing potential variations of growth and product formation by on-line monitoring. BMC Biotechnology, Vol. 10 Nr. 22, Deutschland, 173-183.

Kumar, S., Wittmann, C., Heinzle, E. (2004): Minibioreactors. Biotechnology Letters, Vol. 26 Nr. 1, Deutschland, 1-10.

Mayr, L., Fuerst, P. (2008): The Future of High-Throughput Screening. Journal of Biomolecular Screening, Vol. 13 Nr. 6, Schweiz, 443-448.

Wölcke, J., Ullmann, D. (2001): Miniaturized HTS technologies - uHTS. Drug Discovery Today, Vol. 6 Nr. 12, Deutschland, 637-646.

\section{Autoren}

Ulrich M. Tillich, M. Sc.

Molekularbiologie und funktionelle Genomik Technische Hochschule Wildau [FH]

Stefanie Grüber, B. Sc.

Studentin Biosystemtechnik/Bioinformatik

Technische Hochschule Wildau [FH]

\section{Prof. Dr. Marcus Frohme}

Molekularbiologie und funktionelle Genomik Technische Hochschule Wildau [FH]

marcus.frohme@th-wildau.de 